\title{
Surco diagonal en lóbulo de la oreja. ¿Nos alerta ante un posible cardiópata?
}

\author{
Eva Buller Viqueiraa ${ }^{a}$ Milagros Rocío García Junquero ${ }^{\mathrm{b}}$ y Diego Fernández Martínez ${ }^{\mathrm{b}}$
}

\author{
${ }^{a}$ Médico de Familia. \\ Centro de Salud Gonzalo Pérez \\ Fabra. Paterna de Rivera. \\ Cádiz (España). \\ ${ }^{\mathrm{b}}$ Enfermera. Centro de Salud \\ Gonzalo Pérez Fabra. \\ Paterna de Rivera. \\ Cádiz (España).
}

Correspondencia:

Eva Buller Viqueira.

Centro de Salud Gonzalo Pérez

Fabra. C/ Molino, 14.

C.P. 11178. Paterna de Rivera.

Cádiz (España).

Correo electrónico:

miji_77@yahoo.com.

Recibido el 19 de mayo de 2014. Aceptado para su publicación el 31 de julio de 2014.

\section{RESUMEN}

El signo de Frank consiste en una hendidura diagonal que se inicia en el borde inferior del conducto auricular externo y se dirige con una angulación de $45^{\circ}$ hacia el borde del lóbulo del pabellón auricular. Se ha descrito como marcador de enfermedad coronaria. Presentamos el caso de un varón de 58 años con clínica de larga evolución de dolor precordial y con factores de riesgo cardiovascular, diagnosticado de enfermedad severa de monovaso y en el que se aprecia signo de Frank bilateral.

Palabras Clave. Pabellón Auricular. Enfermedad de la Arteria Coronaria. Oído Externo/ anomalías.

\section{ABSTRACT}

Diagonal crease in the earlobe. Does it alert us about a possible heart disease?

Frank's sign consists of a diagonal crease that starts at the lower border of the external auditory canal and leads to the edge of the ear auricle at a $45^{\circ}$ angle. Described as an indicator of coronary disease The present is a case of a 58 year old man with a long clinical development of chest pain and cardiovascular risk factors, diagnosed with severe damage of a coronary singlevessel and in which we appreciate bilateral Frank's sign.

Key words: Ear Auricle. Coronary Artery Disease. Ear, External/abnormalities.

\section{INTRODUCCIÓN}

El neumólogo Sanders T. Frank asoció el surco diagonal del lóbulo de la oreja con la enfermedad arterial coronaria en 1973 y lo bautizó con el nombre de signo de Frank. El signo de Frank consiste en una hendidura diagonal que se inicia en el borde inferior del conducto auricular externo y se dirige con una angulación de $45^{\circ}$ hacia el borde del lóbulo del pabellón auricular ${ }^{1}$.

Desde su descripción hasta la fecha de hoy existe controversia entre la existencia de este signo y la presencia de cardiopatías, pero sí ha quedado establecida una base genética en relación con el sistema HLA-B27, el gen C3-F de la aterosclerosis y el cromosoma $11^{2}$. Existen estudios que vinculan esta asociación con mayor o menor grado entre los diferentes grupos étnicos, encontrándose que la población japonesa es la de menor incidencia, siendo esta mayor entre los caucásicos, chinos y latinos ${ }^{3}$.

\section{CASO CLÍNICO}

Comentamos el caso de un varón de 58 años con larga evolución de dolor torácico atípico y en cuyo momento presentaba factores de riesgo cardiovascular como hipertensión arterial (HTA), dislipemia e índice de masa corporal de 31 (no fumador, no diabético, no bebedor); además, estaba diagnosticado de depresión. Seguía tratamiento con simvastatina $20 \mathrm{mg}$, bisoprolol $2,5 \mathrm{mg}$, enalapril $20 \mathrm{mg}$, ácido acetilsalicílico (AAS) $100 \mathrm{mg}$, alprazolam 0,5 mg y 
ketazolam $45 \mathrm{mg}$. Acudió a su médico de familia y a urgencias hospitalarias en numerosas ocasiones presentando dolor torácico atípico con electrocardiogramas normales, sin constatar cambios eléctricos ni elevación enzimática. Hace unos 3 años fue valorado por cardiología, presentando una ecocardiografía sin anomalías, con ergometría positiva a alta carga y tomografía computerizada coronaria informada como aterosclerosis coronaria no obstructiva. Tras numerosos episodios, finalmente ingresa para un segundo estudio de posible angina inestable. Se le realizó un cateterismo, visualizando una enfermedad severa de monovaso (en concreto de la arteria circunfleja derecha media), precisando angioplastia coronaria transluminal percutánea e implantación de stent farmacoactivo. En tratamiento actual con nitroglicerina sl si dolor precordial, ramipril 2,5 mg, clopidogrel $75 \mathrm{mg}$, AAS $100 \mathrm{mg}$, simvastatina $40 \mathrm{mg}$, bisoprolol $2,5 \mathrm{mg}$, ranitidina $150 \mathrm{mg}$, ketazolam $45 \mathrm{mg}$ y alprazolam $0,5 \mathrm{mg}$. Tras la colocación del stent, el paciente refiere mejoría clínica.

Posteriormente, en la valoración de consulta de crónicos de enfermería se aprecia de forma casual una hendidura diagonal en el lóbulo del pabellón

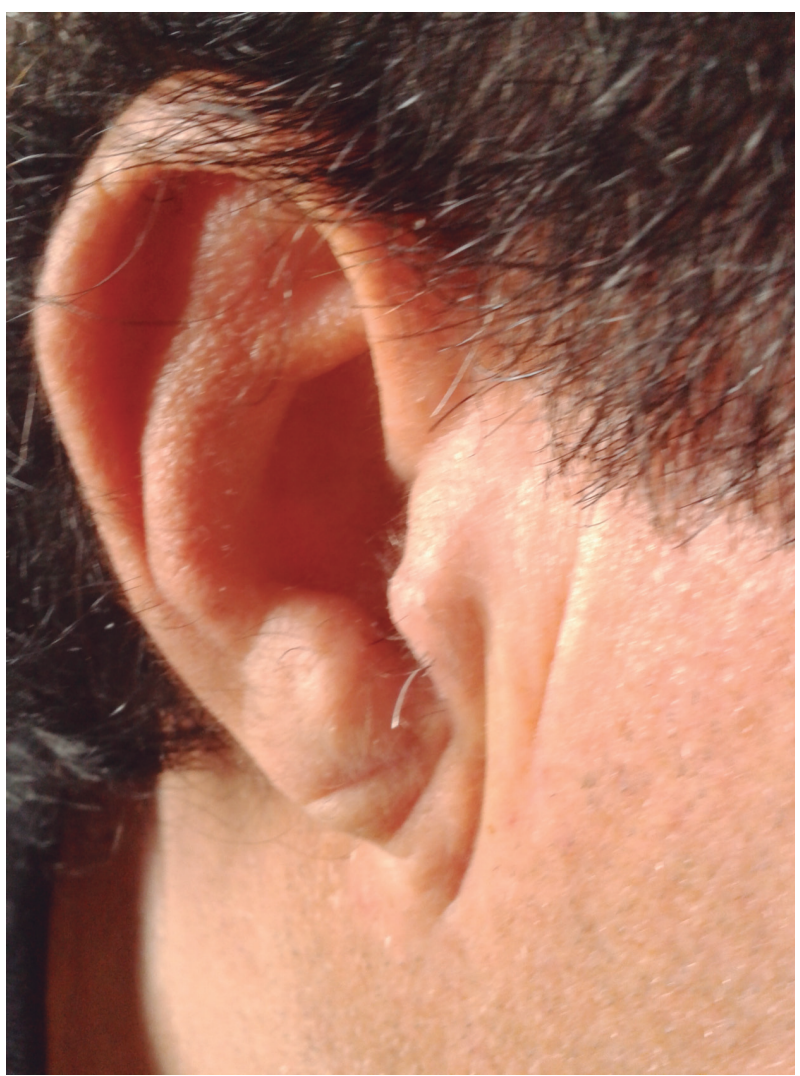

Figura 1. Pabellón auricular con signo de Frank en el paciente caso. auricular que discurre desde la porción anterior y cercano al trago en $45^{\circ}$ hacia la cara posterior del pabellón auricular, bilateral, conocido como signo de Frank (figura 1). Este hallazgo nos hizo pensar que pueda existir una interrelación entre este surco y la cardiopatía diagnosticada.

\section{DISCUSIÓN}

Según algunos autores, la hendidura del lóbulo auricular es más prevalente ante la enfermedad coronaria. Lichstein et al. describen que la presencia del signo de Frank es mayor en pacientes con infarto de miocardio que en los que no lo han sufrido ${ }^{4}$. En otro estudio, al tener en cuenta la enfermedad de la arteria coronaria diagnosticada por angiografía, hubo una mayor asociación estadística en pacientes con la hendidura y un angiograma positivo que entre los que presentaban un angiograma normal ${ }^{5}$. Existen estudios de patólogos forenses que describen una asociación entre la hendidura del lóbulo y la enfermedad coronaria. Varios autores están de acuerdo en que la hendidura es más prevalente a partir de los 50 años y que se relaciona con factores de riesgo cardiovasculares como la obesidad, el tabaquismo y la HTA. Se describe en la literatura la uni o bilateralidad, y la profundidad con la severidad de la lesión ${ }^{6}$ y la mayor asociación con enfermedad coronaria si además de la hendidura hay presencia de pelo en el conducto auditivo externo?

Por lo contrario otros autores no ven significación estadística ante este hallazgo, como Cheng o Koracevic ${ }^{8,9}$, tanto si era uni o bilateral o si la hendidura era profunda o superficial. Existen otros estudios realizados que apoyan esta hipótesis ${ }^{10-12}$. Algunos de los posibles sesgos en los estudios de los que opinan en contra de esta asociación son el haber tomado un tamaño muestral demasiado pequeño, no tener una población control, solo haber seleccionado pacientes con patologías cardíacas, no haber definido de forma concisa los criterios de inclusión para diagnosticar el signo de Frank (uni/ bilateralidad, longitud o profundidad del surco) y no haber tenido en cuenta los grupos étnicos ${ }^{13}$.

Es obvio que aún hay mucho más que estudiar sobre la asociación o no de este signo con la enfermedad coronaria, y dónde mejor se podría hacer este estudio que en Atención Primaria, donde vemos pacientes con y sin cardiopatías, solo tenemos que levantar la vista del ordenador. A nuestro parecer es un signo clínico de fácil diagnóstico y sin coste ni repercusión alguna para el paciente. El signo de Frank puede ser una herramienta de ex- 
ploración clínica, tanto a nivel médico como enfermero. La enfermedad coronaria es algo común en nuestra práctica clínica; qué menos, por tanto, que conocer este "indicador de enfermedad coronaria" para sospechar esta patología ante futuros cardiópatas y en pacientes con sospecha de patología cardiaca como el caso de nuestro paciente para reforzar el diagnostico.

\section{BIBLIOGRAFÍA}

1. Frank ST. Aural sign of coronary-artery disease. $\mathrm{N}$ Engl J Med. 1973; 289 (6): 327-28.

2. Carillo-Esper R, Carrillo-Córdova JR, Carrillo-Córdova LD. Signo del lóbulo hendido, ¿curiosidad clínica o marcador de cardiopatía isquémica? Gac Med Mex, 2010; 146 (3): 225-7.

3. Moncada, B, Ruiz JM, Rodríguez E, Leiva JL. Ear-lobe crease. Lancet. 1979; 1 (8109): 220-1.

4. Lichstein E, Chadda KD, Naik D, Gupta PK. Diagonal earlobe crease: prevalence and implications as a coronary risk factor. N Engl J Med. 1974; 290 (11): 615-6.

5. Evrengül $H$, Dursunoglu $D$, Kaftan A, Soy $M$, Tanriverdi $H$, Zungur $\mathrm{M}$ et al. Bilateral diagonal earlobe crease and coronary artery disease: a significant association. Dermatology. 2004; 209 (4): 271-5.
6. Ishii T, Asuwa N, Masuda S, Ishikawa Y, Shimada K, Talemoto S. Earlobe crease and atherosclerosis. An autopsy study. J Am Geriatr Soc. 1990; 38 (8): 871-6.

7. Wagner RF, Reinfeld HB, Wagner KD, Gambino AT, Falco $\mathrm{TA}$, Sokol JA et al. Ear-canal hair and the ear-lobe crease as predictors for coronary-artery disease. N Engl J Med. 1984; 311 (20): 1317-8.

8. Cheng TO. More research needed on the association between diagonal earlobe crease and coronary artery disease. Arch Intern Med. 2000; 160 (15): 2396-7.

9. Koracevic G, Atanaskovic V. Ear lobe crease: point of disagreement in evidence-based medicine. Am J Forensic Med Pathol. 2009; 30 (1): 89.

10. Jorde LB, Williams RR, Hunt SC. Lack of association of diagonal earlobe creases with other cardiovascular risk factors. West J Med. 1984; 140 (2): 220-3.

11. Motamed M, Pelekoudas N. The predictive value or diagonal ear-lobe crease sign. Int J Clin Pract. 1998; 52 (5): 305-6.

12. Davis TM, Balme M, Jackson D, Sttucio G, Bruce DG. The diagonal earlobe crease (Frank's sign) is not associated with coronary artery disease or retinopathy in type 2 diabetes: the Fremantle Diabetes Study. Aust N Z J Med. 2000; 30 (5): 573-7.

13. Wu XL, Yang DY, Zhao YS, Chai WH, Jin ML. Diagonal earlobe crease and coronary artery disease in a Chinese population. BMC Cardiovasc Disord. 2014; 14: 43. 